\title{
Evaluation of a Metal Artifacts Reduction Algorithm Applied to Postinterventional Flat Panel Detector CT Imaging
}

\author{
D.A. Stidd, H. Theessen, Y. Deng, Y. Li, B. Scholz, C. Rohkohl, M.D. Jhaveri, R. Moftakhar, M. Chen, and D.K. Lopes
}

\begin{abstract}
BACKGROUND AND PURPOSE: Flat panel detector CT images are degraded by streak artifacts caused by radiodense implanted materials such as coils or clips. A new metal artifacts reduction prototype algorithm has been used to minimize these artifacts. The application of this new metal artifacts reduction algorithm was evaluated for flat panel detector CT imaging performed in a routine clinical setting.
\end{abstract}

MATERIALS AND METHODS: Flat panel detector CT images were obtained from 59 patients immediately following cerebral endovascular procedures or as surveillance imaging for cerebral endovascular or surgical procedures previously performed. The images were independently evaluated by 7 physicians for metal artifacts reduction on a 3-point scale at 2 locations: immediately adjacent to the metallic implant and $3 \mathrm{~cm}$ away from it. The number of visible vessels before and after metal artifacts reduction correction was also evaluated within a $3-\mathrm{cm}$ radius around the metallic implant.

RESULTS: The metal artifacts reduction algorithm was applied to the 59 flat panel detector CT datasets without complications. The metal artifacts in the reduction-corrected flat panel detector CT images were significantly reduced in the area immediately adjacent to the implanted metal object $(P=.05)$ and in the area $3 \mathrm{~cm}$ away from the metal object $(P=.03)$. The average number of visible vessel segments increased from 4.07 to $5.29(P=.1235)$ after application of the metal artifacts reduction algorithm to the flat panel detector $C T$ images.

CONCLUSIONS: Metal artifacts reduction is an effective method to improve flat panel detector CT images degraded by metal artifacts. Metal artifacts are significantly decreased by the metal artifacts reduction algorithm, and there was a trend toward increased vesselsegment visualization.

ABBREVIATIONS: $\mathrm{FDCT}=$ flat panel detector $\mathrm{CT} ; \mathrm{MAR}=$ metal artifacts reduction

W ith continued technologic developments, both diagnostic and interventional neuroendovascular applications of flat panel detector CT (FDCT) have increasingly expanded. FDCT offers higher spatial resolution relative to multidetector row CT and improved visualization of clipped aneurysms and endovascular stents. ${ }^{1}$ Imaging performed in the endovascular suite also offers rapid visualization of periprocedural complications, thus allowing faster management. ${ }^{2}$ The acquired FDCT images, however, are degraded by artifacts created by radiodense cerebral endovascular and surgical implants including coils, stents, and clips, limiting the use of FDCT as a postprocedural imaging technique.

Received November 1, 2013; accepted after revision March 20, 2014.

From the Departments of Neurosurgery (D.A.S., R.M., M.C., D.K.L.), Internal Medicine (Y.D., Y.L.), and Radiology (M.D.J.), Rush University Medical Center, Chicago, Illinois; and Siemens Healthcare Sector (B.S., C.R., H.T.), Erlangen, Germany.

Please address correspondence to Demetrius K. Lopes, MD, Rush University Medical Center, 1725 W. Harrison St, Suite 855, Chicago, Illinois 60612; e-mail:

brainaneurysm@mac.com; @Cure4Stroke

http://dx.doi.org/10.3174/ajnr.A4079
Metal artifacts reduction (MAR) in CT images has been attempted by both interpolation and algebraic methods. Interpolation methods have been quite successful and, most important, require less computational power relative to algebraic methods, resulting in less computing time. One of the first interpolationbased MAR algorithms used a 1D linear interpolation for singlerow CT data, which is inadequate for FDCT data. ${ }^{3}$ The MAR algorithm investigated in this work, developed by Siemens Healthcare Sector, is a modification and extension of a recently published MAR procedure by Prell et al. ${ }^{4}$ We present validation of the FDCT MAR prototype algorithm by using a phantom study and an objective critique by 7 clinicians.

\section{MATERIALS AND METHODS Image Acquisition}

During standard clinical care, FDCT images were acquired on a biplane Axiom Artis zee angiography system (Siemens, Erlangen, Germany) by using a 20 -second scan protocol with the following parameters: $70 \mathrm{kV}, 200^{\circ}$ rotation, 496 projections, $1.2 \mu \mathrm{Gy} /$ frame. The average patient radiation dose was 2.9 

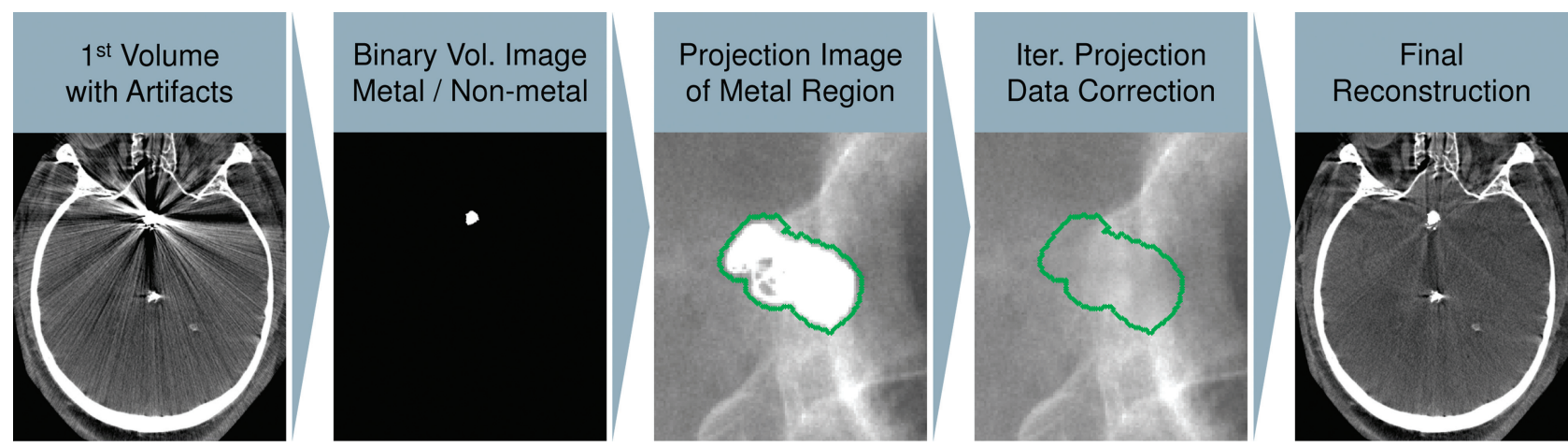

FIG 1. A graphic depiction of the metal artifacts prototype algorithm used for the flat panel detector CT images. An initial volume is reconstructed from the raw data containing metal artifacts. The metallic implant is then segmented, creating a binary volume of the implant, which is forward-projected onto the first reconstruction to identify data corrupted by artifacts. The corrupted data are replaced by a nonlinear interpolation procedure by using the data along the metal region boundaries (depicted in green). A new MAR-corrected volume is reconstructed. The segmented metallic implant is overlaid back onto the dataset for the final reconstruction.

mSv, based on prior measurement data. Twenty-eight datasets were acquired without contrast injection. Of the 31 datasets acquired with contrast, 14 patients received intravenous contrast and 17 patients received intra-arterial contrast. For patients who received IV contrast, $80 \mathrm{~mL}$ was injected at $4 \mathrm{~mL} / \mathrm{s}$, and image acquisition was started after a 14 -second delay. For patients receiving intra-arterial contrast, $40 \mathrm{~mL}$ of $20 \%$ contrast diluted with saline was injected through an intra-arterial catheter at $2 \mathrm{~mL} / \mathrm{s}$. Initial image reconstruction for each case was performed on syngo X Workplace software, Version VB15 or VB21 (Siemens). The parameters used to reconstruct the FDCT images were the following: section thickness, $0.46 \mathrm{~mm}$; "normal" reconstruction kernel; full FOV; $512 \times 512$ matrix, resulting in an isotropic voxel size of $0.3 \mathrm{~mm}^{3}$.

\section{Metal Artifacts Reduction Algorithm}

The MAR prototype algorithm (Fig 1) investigated is a modification and extension of the procedure first published by Prell et $\mathrm{al}^{4}$ The MAR reconstructions were performed on an off-line prototype research workstation (Siemens). The algorithm consists of several steps. Initially, an uncorrected volume image is reconstructed from the acquired imaging data. A binary metal volume image is obtained by segmenting the metallic objects in this volume. In this step, the metallic objects are manually segmented. For each projection, this binary volume is forward-projected to yield a binary projection image of metal regions on the detector in each respective position. The projection data contained in these metal regions are generated by rays through metal objects; thus, these data are responsible for the artifacts. The data along the metal region boundaries are used to replace these data by a nonlinear interpolation procedure. This initial corrected volume is then used for a second normalized MAR correction step. ${ }^{5}$ This step includes additional iterative improvements of the metal region boundaries to enhance the consistency of the corrected data as a whole. Finally, a procedure minimizing the total variation is applied to reduce residual streaks.

\section{Phantom Aneurysm Model}

A phantom aneurysm model was created to demonstrate the reliability and accuracy of the metal artifacts reduction algorithm. The model was created with 10-mm-diameter silicone elastomer tubing with a $12 \times 12 \mathrm{~mm}$ simulated aneurysm. The model was filled with 20\% diluted iopamidol (Isovue 250; Bracco Diagnostics, Princeton, New Jersey) to simulate a patient-like injection protocol and was submerged in a basin of water. A Neuroform EZ Stent (Stryker Neurovascular, Kalamazoo, Michigan) was deployed into the parent vessel of the model, and the aneurysm was filled with 4 Target Detachable Coils (Stryker Neurovascular). A piece of water-soluble polyurethane (Stryker Neurovascular) representative of a clot was placed inside the stent (Fig $2 A$ ). Two sets of FDCT images of the model were obtained in 2 different orthogonal positions relative to the $\mathrm{C}$-arm, by using a 20 -second scan protocol and $109 \mathrm{kV}$. Uncorrected and MAR-corrected volumes were reconstructed as described above (Figs $2 B-E$ ).

\section{Patients}

FDCT scans obtained as routine imaging from 59 patients $(62.7 \%$ women; mean age, 55.9 years; age range, 21-85 years) between January 2012 and May 2013 were retrospectively included as common neuroendovascular images (Figs 3 and 4). Images were selected to be representative of various metal objects in the head and neck region displaying a large amount of metal artifact (Table 1). Images were de-identified, and the MAR algorithm was applied after the conclusion of patient care. This study and imaging protocol were reviewed and approved by the university institutional review board.

\section{Image Evaluation}

The uncorrected and MAR-corrected datasets were independently evaluated by 7 physicians. Two evaluators were dualtrained neurosurgeons practicing both open and neuroendovascular interventions for cerebral vascular disease. One evaluator was a neurologist trained as a neuroendovascular interventionist. Another evaluator was a practicing neuroradiologist. The 3 remaining evaluators were a neurosurgeon training as a fellow in neuroendovascular surgery and 2 radiologists training as fellows in neuroradiology.

The uncorrected datasets of the 59 paired image sets were randomized and evaluated for the amount of metal artifacts present by using a 3-point scale (Table 2) at 2 different locations on the images. The first location was the area immediately surrounding the metallic object, and the second location was an area approx- 

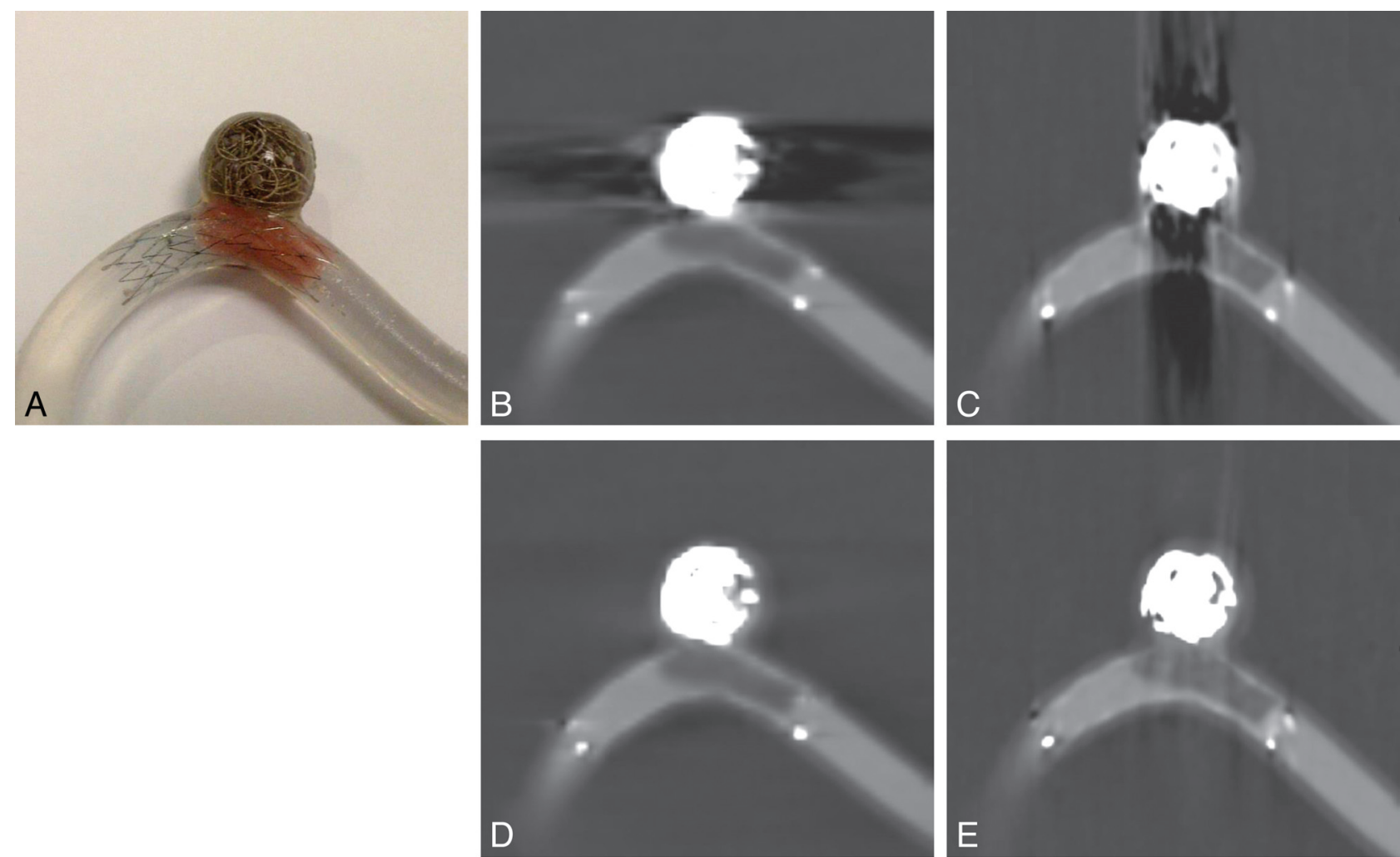

FIG 2. A, A phantom aneurysm model constructed of silicone elastomer tubing used to test the MAR algorithm prototype. Platinum coils were deployed in the aneurysm, and a small piece of polyurethane was placed inside a stent deployed across the aneurysm neck to model a clot. $B$ and C, Uncorrected FDCT images acquired of the model constructed with stent, coils, and the simulated clot show streak artifacts created by the metal alloys of the stent and coils. $D$ and $E$, Corresponding MAR-corrected FDCT images show reduced artifacts.
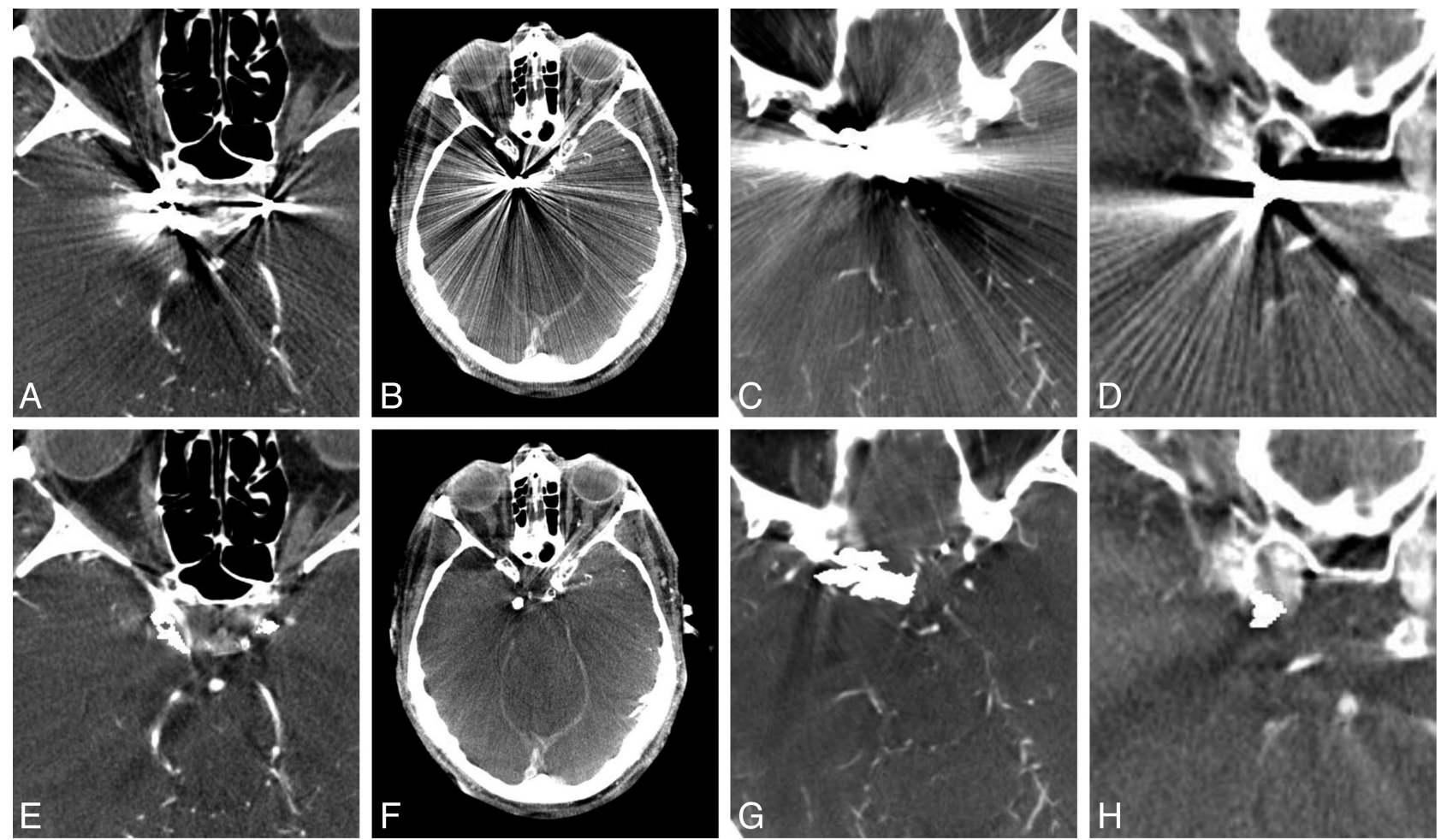

FIG 3. Flat panel detector $C T$ uncorrected $(A-D)$ and corresponding MAR-corrected $(E-H)$ images depicting reduction of streak artifacts caused by coils deployed to treat intracranial aneurysms. The images were independently scored for the amount of metal artifacts and the number of visualized vessel segments within a 3 -cm radius surrounding the metal objects. 

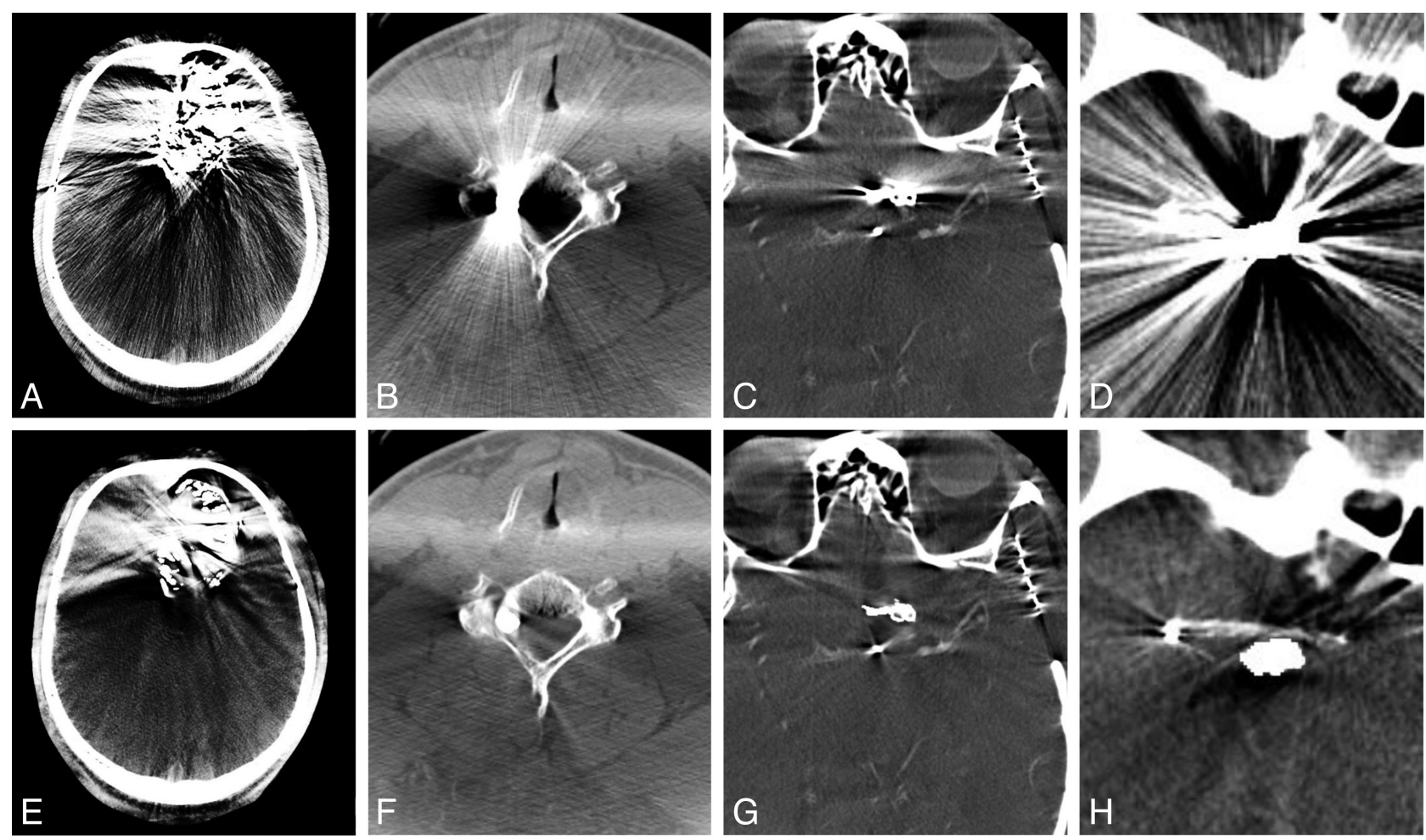

FIG 4. Sample FDCT images of various metallic objects causing streak artifacts and the application of the MAR algorithm. $A$ and $E$, Onyx embolization of an intracranial AVM. The MAR algorithm was less effective at reducing the artifacts caused by Onyx. $B$ and $F$, A bullet lodged within the cervical spine. $C$ and $G, A$ vascular clip used to treat an intracranial aneurysm. $D$ and $H$, Stent-assisted coil embolization of an intracranial aneurysm. This example is similar to the phantom model created to evaluate the MAR algorithm. The stent is completely obscured by the metal artifacts but is visible after the application of the MAR algorithm.

Table 1: Datasets included for MAR evaluation

\begin{tabular}{|c|c|}
\hline Metal Objects & $\begin{array}{c}\text { No. of } \\
\text { Datasets }\end{array}$ \\
\hline Coils only & 19 \\
\hline Clips & 10 \\
\hline Stent & 3 \\
\hline Onyx & 3 \\
\hline Stent and coils & 21 \\
\hline Other (bullet, spinal screws, mandibular fixtures) & 3 \\
\hline
\end{tabular}

Table 2: Score used to quantify the amount of metal artifacts

\begin{tabular}{cc}
\hline Score & Definition \\
\hline 0 & $\begin{array}{c}\text { No metal artifacts; relevant surrounding anatomy } \\
\text { well-visualized }\end{array}$ \\
1 & $\begin{array}{c}\text { Moderate metal artifacts; relevant anatomy visible but } \\
\text { affected by artifacts }\end{array}$ \\
2 & Severe artifacts; relevant anatomy not visible \\
\hline
\end{tabular}

imately $3 \mathrm{~cm}$ away from the metallic object that was affected the most by streak artifacts. The 59 MAR-corrected datasets were evaluated in a similar manner. The scores assigned by the evaluators were recorded for analysis later. All images were evaluated at an imaging workstation with a medical-grade monitor (Siemens). Each evaluator had the ability to adjust image contrast and brightness levels and scroll through the topography sections as needed.

Of the 31 datasets acquired with contrast, 25 image sets were selected as representative acquisitions of cerebral vasculature. A single section through the metallic object most degraded by arti- facts was selected for each uncorrected dataset, and the corresponding section of the MAR-corrected dataset was selected. These 50 images were randomized and loaded onto a viewer. A circle centered on the metallic object defining an area with a radius of $3 \mathrm{~cm}$ was overlaid onto each image. The 7 evaluators then independently counted the number of visible vessel segments within the defined areas without the ability to adjust contrast or brightness levels. The number of vessels counted for each image was recorded for analysis later. Bias was minimized by randomizing the 25 pairs of prepared images, creating 50 separate images presented in random order.

\section{Statistical Analysis}

The amount of metal artifacts rated on a 3-point scale for the images were reported as relative frequencies. Nonparametric paired Wilcoxon signed rank tests were used to test for statistically significant differences among the distributions of scored artifacts values. The results of the vessel-count analysis were reported as means \pm standard error of the mean, and paired $t$ tests were used for statistically significant differences between the uncorrected and corrected groups. $P$ values were calculated as 2 -tailed; $P<.05$ was considered statistically significant. Interobserver agreement was measured for the metal artifacts scoring by calculating the $\kappa$ statistic and interpreting the results as suggested by Landis and Koch. ${ }^{6}$ A 1-way absolute-agreement intraclass correlation coefficient was used to assess the interobserver agreement for the vesselcount analysis. All statistical analyses were conducted by using the STATA 11 statistical software package (StataCorp, College Sta- 


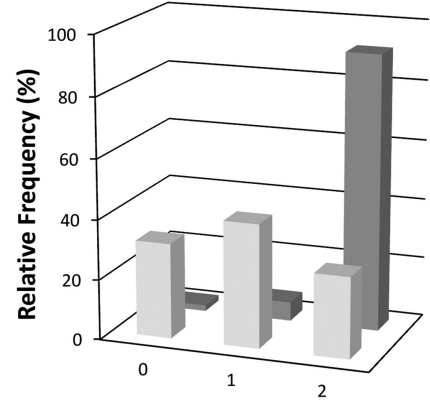

A

Metal Artifact Score

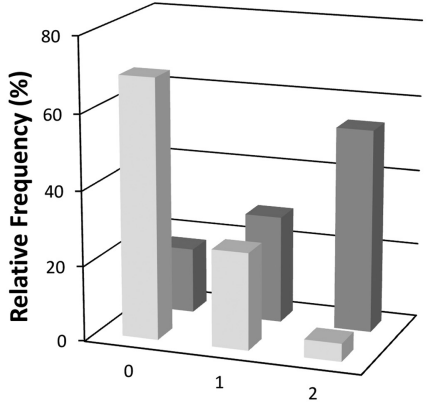

B Metal Artifact Score

FIG 5. Relative frequencies of metal artifacts scores assigned to FDCT scans for areas immediately adjacent to the implanted metal object $(A)$ or $3 \mathrm{~cm}$ away from the implanted metal object $(B)$. The median $P$ value for the uncorrected and MAR-corrected image pairs adjacent to the metal object was $P=.05$, and the median $P$ value for the image pairs $3 \mathrm{~cm}$ away was $P=.03$. The uncorrected and MAR-corrected images were independently evaluated by 7 clinicians on a 3-point scale $(n=59)$.

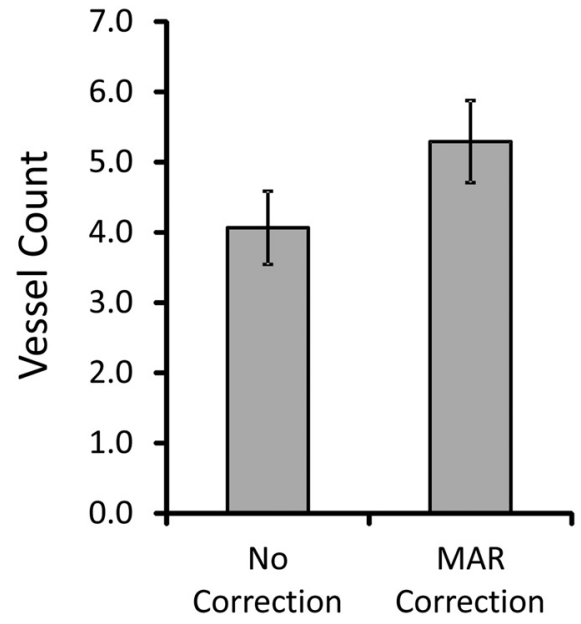

FIG 6. The mean number of visualized vessel segments within a $30-\mathrm{cm}$ radius of the implanted metal object before and after MAR correction. The images were independently evaluated by 7 clinicians. The error bars represent the standard error of the mean $(n=25)$.

tion, Texas) or the package "irr" of $\mathrm{R}$, Version 0.84 (http://www.r-project.org/).

\section{RESULTS}

Metal streak artifacts were noted on the acquired FDCT images of the phantom aneurysm model (Fig $2 B,-C$ ). The artifacts were caused by the metal alloys contained within the stent and coils, and the artifacts were directed in-line to the radiation beam. Application of the MAR algorithm qualitatively reduced the amount of streak artifacts without corresponding image degradation (Fig $2 D,-E)$. The simulated polyurethane clot within the model filled with $20 \%$ contrast was visible as a hypoattenuation at the level of the stent. The visibility of the clot remained qualitatively unchanged after the application of the MAR algorithm. The space not occupied by coils within the aneurysm was notably more defined.

The MAR algorithm was applied to the 59 acquired patient FDCT scans with metallic implants without difficulty, and the uncorrected and MAR-corrected datasets were evaluated for metal artifacts (Fig 5). A 3-point scale was used to quantify the amount of metal artifacts (Table 2). The relative frequencies of the

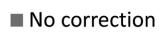

MAR correction quantified amount of metal artifacts adjacent to the metallic implants scored as not present, moderate, or severe were, respectively, $1.9 \%, 6.3 \%$, and $91.8 \%$ for the uncorrected images and $31.7 \%$, $41.2 \%$, and $27.1 \%$ for the MAR-corrected images (median $P=.05$ ). In an area $3 \mathrm{~cm}$ away from the metallic implants, the relative frequencies of the quantified metal artifacts scored as not present, moderate, or severe were, respectively, $17.4 \%, 28.6 \%$, and $54 \%$ for the uncorrected images and $69.2 \%$, $25.9 \%$, and $4.8 \%$ for the MAR-corrected images (median $P=.03$ ). There was moderate interobserver agreement among the 7 evaluators $(\kappa=0.44)$. The MAR algorithm subjectively was not as effective in the 3 datasets involving Onyx (Covidien, Irvine, California). When analyzed separately, the relative frequencies of the quantified amount of metal artifacts adjacent to the Onyx cast scored as not present, moderate, or severe were respectively $0 \%$, $4.8 \%$, and $95.2 \%$ for the uncorrected images and $0 \%, 52.4 \%$, and $47.6 \%$ for the MAR-corrected images (median $P=.13$ ). At $3 \mathrm{~cm}$ away from the Onyx cast, the relative frequencies of the amount of artifacts scored as not present, moderate, or severe were, respectively, $23.8 \%, 19.0 \%$, and $57.1 \%$ for the uncorrected images and $52.4 \%, 19.0 \%$, and $28.6 \%$ for the MAR-corrected images (median $P=1.00)$.

Twenty-five pairs of uncorrected and MAR-corrected image sets were evaluated for the number of visible vessel segments within a $3-\mathrm{cm}$ radius of the metallic implant (Fig 6). The average number of visible vessel segments increased from $4.07 \pm 0.52$ to $5.29 \pm 0.58(P=.124)$ after the MAR algorithm was applied to the images. There was moderate interobserver agreement for the vessel count analysis (intraclass correlation coefficient $=0.55$ ).

\section{DISCUSSION}

Late detection and management of complications occurring after neuroendovascular procedures have an adverse impact on patient outcome. ${ }^{7-9}$ Transporting a patient to a conventional multidetector CT scan after a complication delays critical decision-making and time to intervention. Technical advances in both image acquisition and postprocessing have improved FDCT imaging quality. Relative to multidetector CT imaging, FDCT imaging has better spatial resolution, ${ }^{1,10}$ making it better suited to visualize the fine geometric details of the metal stents and coils used in neuroendovascular interventional procedures ${ }^{11}$ and fine cerebral vascular anatomy. ${ }^{12}$ The metal alloys within the implants, however, degrade the image quality of FDCT imaging, limiting its use as a routine postprocedural technique. An effective means of reducing the metal artifacts caused by metal implants would make FDCT imaging an ideal postprocedural technique to visualize complications such as intracranial hemorrhage, residual filling of aneurysms, or thrombus within newly deployed stents.

Several strategies have been proposed to reduce the metal artifacts for conventional multidetector row $\mathrm{CT},{ }^{13}$ but these strate- 
gies cannot be directly applied to images acquired with a flat panel detector. The first FDCT-adequate MAR algorithm proposed by Prell et $\mathrm{al}^{2}$ used a nonlinear interpolation procedure to restore corrupted data and has subsequently been shown to decrease metal artifacts of FDCT images obtained for neuroendovascular procedures. ${ }^{13,14}$ This finding correlates with the results of the current study in which we show that the MAR algorithm described above significantly lowered the amount of metal artifacts hindering visualization of the intracranial tissues. Another strategy used to successfully reduce metal artifacts in FDCT imaging is to subtract a volume reconstructed from data corrupted by artifacts from an initial uncorrected volume reconstruction without a nonlinear interpolation process to replace the corrupted data. ${ }^{15}$

The mean number of visualized vessel segments within a $3-\mathrm{cm}$ radius centered on the metallic foreign body was increased after the MAR algorithm was applied, though this was not a significant increase. This lack of significance is likely due to the decreased interobserver agreement. In this study, 7 independent evaluators were used from 3 different subspecialties, hence reflecting a variety of training styles and backgrounds.

As demonstrated in Fig $4 A,-E$, the MAR algorithm prototype was not as effective at reducing the metal streak artifacts caused by the tantalum within the Onyx in datasets of liquid embolization. Onyx casts are very inhomogeneous structures with various densities; therefore, it becomes difficult to determine all boundaries precisely. This imprecise boundary definition manifests as a nonoptimal interpolation result, which likely induces residual streaks in the newly reconstructed volume. The MAR prototype algorithm will need further improvement to minimize streak artifacts caused by Onyx.

The MAR algorithm used for this study did reduce the amount of metal artifacts in all datasets, but the corrected images with reduced streak artifacts may not have improved diagnostic information. The corrupted data replaced by the nonlinear interpolation process may contain new subtle artifacts that might obscure data in the original acquisition. Also, data that are completely degraded by severe artifacts, including motion artifacts or conebeam artifacts at the skull base, for example, cannot be recovered by the MAR algorithm. ${ }^{13}$ Despite these limitations, the MAR algorithm represents a significant improvement of the FDCT image quality, increasing the value of FDCT as a post-neurointerventional procedure imaging technique.

\section{CONCLUSIONS}

The evaluated MAR algorithm is effective in improving FDCT images degraded by streak artifacts caused by metallic implants used for cerebral endovascular and open interventions. Metal artifacts are significantly decreased by the MAR algorithm, with a trend toward increased vessel-segment visualization. These decreased metal artifacts increase the chance for visualizing complications associated with cerebrovascular interventions such as acute hemorrhage surrounding a coiled aneurysm and increase the value of FDCT as a source of postprocedural imaging.

\section{ACKNOWLEDGMENTS}

We recognize Drs Arthur Maydell and Sumeet G. Dua for their contribution to this project.

Disclosures: Heike Theessen, Bernhard Scholz, and Christopher Rohkohl—RELATED: full-time employees of Siemens Healthcare Sector. Miral D. Jhaveri-UNRELATED: Royalties: Amirsys. Demetrius K. Lopes-UNRELATED: Consultancy: Covidien, Stryker, Penumbra, Siemens*; Grants/Grants Pending: Siemens*; Payment for Development of Educational Presentations: Covidien, Penumbra; Stock/Stock Options: Penumbra, VasSol, Blockade Medical; Travel/Accommodations/Meeting Expenses Unrelated to Activities Listed: Siemens. * *Money paid to the institution.

\section{REFERENCES}

1. Gupta R, Cheung AC, Bartling SH, et al. Flat-panel volume CT: fundamental principles, technology, and applications. Radiographics 2008;28:2009-22

2. Prell D, Kyriakou Y, Struffert T, et al. Metal artifact reduction for clipping and coiling in interventional C-arm CT. AJNR Am J Neuroradiol 2010;31:634-39

3. Kalender WA, Hebel R, Ebersberger J. Reduction of CT artifacts caused by metallic implants. Radiology 1987;164:576-77

4. Prell D, Kyriakou Y, Beister M, et al. A novel forward projectionbased metal artifact reduction method for flat-detector computed tomography. Phys Med Biol 2009;54:6575-91

5. Meyer E, Raupach R, Lell M, et al. Normalized metal artifact reduction (NMAR) in computed tomography. Med Phys 2010;37:5482-93

6. Landis JR, Koch GG. The measurement of observer agreement for categorical data. Biometrics 1977;33:159-74

7. Horowitz MB, Crammond D, Balzer J, et al. Aneurysm rupture during endovascular coiling: effects on cerebral transit time and neurophysiologic monitoring and the benefits of early ventriculostomy: case report. Minim Invasive Neurosurg 2003;46:300-05

8. Levy E, Koebbe CJ, Horowitz MB, et al. Rupture of intracranial aneurysms during endovascular coiling: management and outcomes. Neurosurgery 2001;49:807-11, discussion 811-13

9. Cloft HJ, Kallmes DF. Cerebral aneurysm perforations complicating therapy with Guglielmi detachable coils: a meta-analysis. AJNR Am J Neuroradiol 2002;23:1706-09

10. Heran NS, Song JK, Namba K, et al. The utility of DynaCT in neuroendovascular procedures. AJNR Am J Neuroradiol 2006;27:330-32

11. Ionescu M, Metcalfe RW, Cody D, et al. Spatial resolution limits of multislice computed tomography (MS-CT), C-arm-CT, and flat panel-CT (FP-CT) compared to MicroCT for visualization of a small metallic stent. Acad Radiol 2011;18:866-75

12. Saake M, Goelitz P, Struffert T, et al. Comparison of conventional CTA and volume perfusion CTA in evaluation of cerebral arterial vasculature in acute stroke. AJNR Am J Neuroradiol 2012;33:2068-73

13. van der Bom IM, Hou SY, Puri AS, et al. Reduction of coil mass artifacts in high-resolution flat detector conebeam CT of cerebral stent-assisted coiling. AJNR Am J Neuroradiol 2013;34:2163-70

14. Psychogios MN, Scholz B, Rohkohl C, et al. Impact of a new metal artefact reduction algorithm in the noninvasive follow-up of intracranial clips, coils, and stents with flat-panel angiographic CTA: initial results. Neuroradiology 2013;55:813-18

15. Buhk JH, Groth M, Sehner S, et al. Application of a novel metal artifact correction algorithm in flat-panel CT after coil embolization of brain aneurysms: intraindividual comparison. Rofo 2013; $185: 824-29$ 